\title{
EXISTENCE AND MULTIPLICITY OF SOLUTIONS FOR SOME THREE-POINT NONLINEAR BOUNDARY VALUE PROBLEMS
}

XU XIAN AND DONAL O'REGAN

Received 30 September 2004; Accepted 20 October 2004

We study the existence and multiplicity of solutions for the three-point nonlinear boundary value problem $u^{\prime \prime}(t)+\lambda a(t) f(u)=0,0<t<1 ; u(0)=0=u(1)-\gamma u(\eta)$, where $\eta \in$ $(0,1), \gamma \in[0,1), a(t)$ and $f(u)$ are assumed to be positive and have some singularities, and $\lambda$ is a positive parameter. Under certain conditions, we prove that there exists $\lambda^{*}>0$ such that the three-point nonlinear boundary value problem has at least two positive solutions for $0<\lambda<\lambda^{*}$, at least one solution for $\lambda=\lambda^{*}$, and no solution for $\lambda>\lambda^{*}$.

Copyright (C 2006 X. Xian and D. O’Regan. This is an open access article distributed under the Creative Commons Attribution License, which permits unrestricted use, distribution, and reproduction in any medium, provided the original work is properly cited.

\section{Introduction}

In this paper, we consider the following second-order three-point boundary value problem (BVP)

$$
\begin{gathered}
u^{\prime \prime}(t)+\lambda a(t) f(u)=0, \quad 0<t<1, \\
u(0)=0=u(1)-\gamma u(\eta),
\end{gathered}
$$

where $\eta \in(0,1), \gamma \in[0,1), a \in C((0,1),(0,+\infty))$, and $f \in C\left(\mathbb{R}^{+} \backslash\{0\}, \mathbb{R}^{+}\right)$, here $\lambda$ is a positive parameter and $\mathbb{R}^{+}=[0,+\infty)$.

Now $a(t)$ may have a singularity at $t=0$ and $t=1, f(u)$ may have a singularity at $u=0$, so the BVP $\left(1.1_{\lambda}\right)$ is a singular problem. The BVP $\left(1.1_{\lambda}\right)$ in the case when $\gamma=0$ can be reduced to the Dirichlet BVP

$$
\begin{aligned}
u^{\prime \prime}(t)+\lambda a(t) f(u) & =0, \quad 0<t<1, \\
u(0)=0 & =u(1) .
\end{aligned}
$$

The BVP $\left(1.2_{\lambda}\right)$ has been studied extensively in the literature, see $[1,2,5,9,12]$ and the references therein. Choi [1] studied the particular case where $f(u)=e^{u}, a \in C^{1}(0,1]$, $a>0$ in $(0,1)$, and $a$ can be singular at $t=0$, but is at most $O\left(1 / t^{2-\delta}\right)$ as $t \rightarrow 0^{+}$for some $\delta$. Using the shooting method, he established the following result. 
Theorem 1.1 (see [1]). There exists $\lambda_{0}>0$ such that the BVP $\left(1.2_{\lambda}\right)$ has a solution in $C^{2}(0,1] \cap C[0,1]$ for $0<\lambda<\lambda_{0}$, while there is no solution for $\lambda>\lambda_{0}$.

Wong [9] studied the more general BVP $\left(1.2_{\lambda}\right)$. Using also the shooting method, Wong proved some existence results for positive solutions of the BVP $\left(1.2_{\lambda}\right)$. Recently, Dalmasso [2] improved Theorem 1.1 and the main results in [9]. Using the upper and lower solutions technique and the fixed point index method, Dalmasso [2] proved the following result.

Theorem 1.2 (see [2]). Let $a$ and $f$ satisfy the following assumptions:

$\left(\mathrm{A}_{1}\right) a \in C((0,1),[0, \infty)), a \neq 0$ in $(0,1)$, and there exists $\alpha, \beta \in[0,1)$ such that

$$
\int_{0}^{1} s^{\alpha}(1-s)^{\beta} a(s) d s<\infty
$$

$\left(\mathrm{A}_{2}\right) f \in C([0, \infty),(0, \infty))$ is nondecreasing.

Then,

(i) there exists $\lambda_{0}>0$ such that the BVP $\left(1.2_{\lambda}\right)$ has at least one positive solution in $C^{2}(0,1) \cap C[0,1]$ for $0<\lambda<\lambda_{0}$,

(ii) if in addition $f$ satisfies the condition that

$\left(\mathrm{A}_{3}\right)$ there exists $d>0$ such that $f(u) \geq d u$ for $u \geq 0$.

Then there exists $\lambda^{*}>0$ such that the BVP $\left(1.2_{\lambda}\right)$ has at least one positive solution in $C^{2}(0$, 1) $\cap C[0,1]$ for $0<\lambda<\lambda^{*}$ while there is no such solution for $\lambda>\lambda^{*}$.

Ha and Lee [5] also considered the BVP $\left(1.2_{\lambda}\right)$ in the case when $f(u) \geq e^{u}$. They proved Theorems 1.3 and 1.4 .

Theorem 1.3 (see [5]). Assume the following conditions hold

$\left(\mathrm{B}_{1}\right)$ a $>0$ on $(0,1)$;

$\left(\mathrm{B}_{2}\right) a(t)$ is singular at $t=0$ satisfying $\int_{0}^{1} s a(s) d s<\infty$;

$\left(\mathrm{B}_{3}\right) f(u) \geq e^{u}$ for all $u \in \mathbb{R}$.

Then there exists $\lambda_{0}$ such that the BVP $\left(1.2_{\lambda}\right)$ has no solution for $\lambda>\lambda_{0}$ and at least one solution for $0<\lambda<\lambda_{0}$.

Theorem 1.4 (see [5]). Consider $\left(1.2_{\lambda}\right)$, where $a$ and $f$ are continuous and satisfy $\left(B_{1}\right)$ $\left(B_{3}\right)$. Also assume that

$\left(\mathrm{B}_{4}\right) f$ is nondecreasing.

Then the number $\lambda_{0}$ given by Theorem 1.3 is such that

(i) $\left(1.2_{\lambda}\right)$ has no solution for $\lambda>\lambda_{0}$;

(ii) $\left(1.2_{\lambda}\right)$ has at least one solution for $\lambda=\lambda_{0}$;

(iii) $\left(1.2_{\lambda}\right)$ has at least two solutions for $0<\lambda<\lambda_{0}$.

$\mathrm{Xu}$ and $\mathrm{Ma}[12]$ generalized the main results of $[1,2,5,9]$ to an operator equation in a real Banach space $E$. In recent years, the multipoint BVP has been extensively studied (see $[3,4,6-8,10,11,13]$ and the references therein). For example, Ma and Castaneda [7] using the well-known fixed point theorem in cones established some results on the existence of at least one positive solution for some $m$-point boundary value problems if the nonlinearity $f$ is either superlinear or sublinear. The purpose of this paper is to 
extend the main results of $[1,2,5,9]$ to the nonlinear three-point BVP $\left(1.1_{\lambda}\right)$. We will consider the existence and multiplicity of positive solution for the nonlinear three-point BVP $\left(1.1_{\lambda}\right)$. The results of this paper are improvements of the main results in $[1,2,5,9]$.

\section{Several lemmas}

Let us list some conditions to be used in this paper.

$\left(\mathrm{H}_{1}\right) \gamma \in[0,1), a \in C((0,1),(0, \infty))$, and

$$
\int_{0}^{1} s(1-s) a(s) d s<\infty
$$

$\left(\mathrm{H}_{2}\right) f(u)=g(u)+h(u)$, where $g:(0, \infty) \mapsto(0, \infty)$ is continuous and nonincreasing, $h: \mathbb{R}^{+} \mapsto \mathbb{R}^{+}$is continuous, and

$$
h(u) \geq b_{0} u^{w}, \quad u \in \mathbb{R}^{+},
$$

for some $b_{0}>0$ and $w \geq 1$.

$\left(\mathrm{H}_{3}\right)$ There exists $M>0$ such that

$$
h\left(u_{2}\right)-h\left(u_{1}\right) \geq-M\left(u_{2}-u_{1}\right)
$$

for all $u_{1}, u_{2} \in \mathbb{R}^{+}$with $u_{2} \geq u_{1}$

The main results of this paper are the following theorems.

Theorem 2.1. Assume that $\left(H_{1}\right)$ and $\left(H_{2}\right)$ hold. Then there exists $\lambda^{*}>0$ such that the BVP $\left(1.1_{\lambda}\right)$ has at least one positive solution for $0<\lambda<\lambda^{*}$ and no solution for $\lambda>\lambda^{*}$.

Moreover, the BVP $\left(1.1_{\lambda}\right)$ has at least one positive solution if $\omega>1$.

Theorem 2.2. Assume that $\left(H_{1}\right),\left(H_{2}\right)$, and $\left(H_{3}\right)$ hold, $\omega>1$, and there exists constant $c \geq 0$ such that $g(u)=c$ for all $u \in(0,+\infty)$. Then there exists $\lambda^{*}>0$ such that the BVP $\left(1.1_{\lambda}\right)$ has at least two positive solutions for $0<\lambda<\lambda^{*}$, at least one solution for $\lambda=\lambda^{*}$, and no solution for $\lambda>\lambda^{*}$.

Remark 2.3. Our theorems generalize Theorems 1.1-1.4 and the main results in [9]. In fact, Theorems 1.1-1.4 are corollaries of our theorems. Moreover, the nonlinear term $f(u)$ may have singularity at $u=0$, therefore, even in the case when $\gamma=0$, Theorem 2.1 cannot be obtained by Theorems 1.1-1.4 and the abstract results in [12].

Remark 2.4. The nonlinear term $f$ was assumed to be nondecreasing in Theorems 1.2 and 1.4, but in Theorem 2.2 in this paper, we do not assume that the nonlinear term $f$ is nondecreasing. Thus, even in the case when $\gamma=0$, Theorem 2.2 cannot be obtained from Theorem 1.4.

Let $n \in \mathbb{N}$ and let $\mathbb{N}$ be the natural numbers set. First, let us consider the BVP of the form

$$
\begin{gathered}
u^{\prime \prime}(t)+\lambda a(t)\left(g\left(u+\frac{1}{n}\right)+h(u)\right)=0, \quad 0<t<1, \\
u(0)=0=u(1)-\gamma u(\eta) .
\end{gathered}
$$


4 Solutions of three-point nonlinear BVPs

Definition 2.5. $\alpha \in C([0,1], \mathbb{R}) \cap C^{2}((0,1), \mathbb{R})$ is called a lower solution of $\left(2.1_{n}^{\lambda}\right)$ if

$$
\begin{gathered}
\alpha^{\prime \prime}(t)+\lambda a(t)\left(g\left(\alpha(t)+\frac{1}{n}\right)+h(\alpha(t))\right) \geq 0, \quad t \in(0,1), \\
\alpha(0) \leq 0, \quad \alpha(1)-\gamma \alpha(\eta) \leq 0 .
\end{gathered}
$$

$\beta \in C([0,1], \mathbb{R}) \cap C^{2}((0,1), \mathbb{R})$ is called an upper solution of $\left(2.1_{n}^{\lambda}\right)$ if

$$
\begin{gathered}
\beta^{\prime \prime}(t)+\lambda a(t)\left(g\left(\beta(t)+\frac{1}{n}\right)+h(\beta(t))\right) \leq 0, \quad t \in(0,1), \\
\beta(0) \geq 0, \quad \beta(1)-\gamma \beta(\eta) \geq 0 .
\end{gathered}
$$

According to [13, Lemma 4], we have the following lemma.

Lemma 2.6. Assume that $\left(H_{1}\right)$ holds and $\tau \geq 0$. Then the initial value problems

$$
\begin{gathered}
u^{\prime \prime}(t)=\tau a(t) u(t), \quad 0 \leq \alpha<t<1, \\
u(\alpha)=0, \quad u^{\prime}(\alpha)=1, \\
u^{\prime \prime}(t)=\tau a(t) u(t), \quad 0<t<\beta \leq 1, \\
u(\beta)=0, \quad u^{\prime}(\beta)=-1
\end{gathered}
$$

have unique positive solutions $p_{\alpha, \tau}(t) \in A C[\alpha, 1) \cap C^{1}[\alpha, 1)$ and $q_{\beta, \tau}(t) \in A C(0, \beta] \cap$ $C^{1}(0, \beta]$, respectively. Moreover, $p_{\alpha, \tau}$ and $q_{\beta, \tau}$ are strictly convex. As a result,

$$
\begin{array}{ll}
t-\alpha \leq p_{\alpha, \tau}(t) \leq p_{\alpha, \tau}(a) \frac{(t-\alpha)}{(a-\alpha)}, & \alpha \leq t \leq a \leq 1, \\
\beta-t \leq q_{\beta, \tau}(t) \leq q_{\beta, \tau}(b) \frac{(\beta-t)}{(\beta-b)}, & 0 \leq b \leq t \leq \beta
\end{array}
$$

for any $a \in[\alpha, 1)$ and $b \in[0, \beta)$.

When $0 \leq \alpha<\beta \leq 1$, for $t \in[\alpha, \beta]$,

$$
W_{[\alpha, \beta]}^{(\tau)}(t)=\left|\begin{array}{ll}
q_{\beta, \tau}(t), & p_{\alpha, \tau}(t) \\
q_{\beta, \tau}^{\prime}(t), & p_{\alpha, \tau}^{\prime}(t)
\end{array}\right|=q_{\beta, \tau}(\alpha)=p_{\alpha, \tau}(\beta) .
$$

It is well known that $C[0,1]$ is a Banach space with maximum norm $\|\cdot\|$. For $\tau \geq 0$, denote $\theta_{\tau}$ by

$$
\theta_{\tau}=\frac{\gamma(1-\eta)}{p_{0, \tau}(\eta)+q_{1, \tau}(\eta)} \min \left\{\frac{p_{0, \tau}(\eta)}{p_{0, \tau}(1)+p_{0, \tau}(\eta)}, \frac{q_{1, \tau}(\eta)}{q_{1, \tau}(0)+q_{1, \tau}(\eta)}\right\} .
$$


Let $P=\{x \in C[0,1] \mid x(t) \geq 0$ for $t \in[0,1]\}$ and $Q_{\tau}=\left\{x \in P \mid x(t) \geq \theta_{\tau}\|x\| t\right.$ for $\left.t \in[0,1]\right\}$. It is easy to see that $P$ and $Q_{\tau}$ are cones in $C[0,1]$. For $\tau \geq 0$ and each $n \in \mathbb{N}$, define operators $L_{\tau}$ and $F_{n}: C[0,1] \mapsto C[0,1]$ by

$$
\left(L_{\tau} x\right)(t)= \begin{cases}\frac{p_{0, \tau}(1)}{p_{0, \tau}(1)-\gamma p_{0, \tau}(\eta)} \int_{0}^{1} G_{[0,1]}^{(\tau)}(\eta, s) a(s) x(s) d s, & t=\eta, \\ \int_{0}^{\eta} G_{[0, \eta]}^{(\tau)}(t, s) a(s) x(s) d s+\left(L_{\tau} x\right)(\eta) \frac{p_{0, \tau}(t)}{p_{0, \tau}(\eta)}, & t \in[0, \eta], \\ \int_{\eta}^{1} G_{[\eta, 1]}^{(\tau)}(t, s) a(s) x(s) d s+\left(L_{\tau} x\right)(\eta) \frac{q_{1, \tau}(t)+\gamma p_{\eta, \tau}(t)}{q_{1, \tau}(\eta)}, & t \in[\eta, 1],\end{cases}
$$

and $\left(F_{n} x\right)(t)=g(x(t)+1 / n)+h(x(t))$ for $t \in[0,1]$, where

$$
G_{[\alpha, \beta]}^{(\tau)}(t, s):= \begin{cases}q_{\beta, \tau}(t) \frac{p_{\alpha, \tau}(s)}{p_{\alpha, \tau}(\beta)}, & \alpha \leq s \leq t \leq \beta, \\ p_{\alpha, \tau}(t) \frac{q_{\beta, \tau}(s)}{q_{\beta, \tau}(\alpha)}, & \alpha \leq t \leq s \leq \beta .\end{cases}
$$

From [13, Theorem 5], we have Lemmas 2.7 and 2.9.

Lemma 2.7. Assume that $\left(H_{1}\right)$ holds, $\tau \geq 0$, and $h \in C([0,1], R)$. Then $w(t)$ is the solution of the three-point BVP

$$
\begin{gathered}
-w^{\prime \prime}(t)+\tau a(t) w(t)=a(t) h(t), \quad 0 \leq \alpha<t \leq 1, \\
w(\alpha)=0=w(1)-\gamma w(\eta)
\end{gathered}
$$

if and only if $w \in C[0,1]$ is the solution of the integral equation

$$
w(t)=\left(L_{\tau} h\right)(t), \quad t \in[0,1] .
$$

Remark 2.8. To ensure that $p_{\alpha, \tau}(1)-\gamma p_{\alpha, \tau}(\eta)>0$, the following condition is assumed in [13, Theorem 5]:

$$
\tau a(t)>\frac{3 \gamma}{(1-\eta)^{2}}
$$

If $0 \leq \gamma<1$, we have

$$
p_{\alpha, \tau}(1)-\gamma p_{\alpha, \tau}(\eta)>p_{\alpha, \tau}(\eta)\left(1+\int_{\eta}^{1} \tau a(s) q_{1, \tau}(s) d s-\gamma\right)>0
$$

Thus, if $0 \leq \gamma<1$, condition (2.14) can be removed. 
6 Solutions of three-point nonlinear BVPs

Lemma 2.9. Assume that $\left(H_{1}\right)$ holds, $\tau, \alpha, \xi^{*}, \eta^{*} \geq 0, h \in C\left([0,1], \mathbb{R}^{+}\right)$. Also suppose that $w \in C[\alpha, 1]$ satisfies

$$
\begin{gathered}
-w^{\prime \prime}(t)+\tau a(t) w(t)=a(t) h(t), \quad \alpha<t<1, \\
w(\alpha)=\xi^{*}, \quad w(1)-\gamma w(\eta)=\eta^{*} .
\end{gathered}
$$

Then $w(t) \geq 0$ for $t \in[\alpha, 1]$.

Lemma 2.10. Assume that $\left(H_{1}\right)$ holds and $\tau \geq 0$. Then $L_{\tau}: P \mapsto Q_{\tau}$ is a completely continuous and increasing operator.

Proof. From Lemma 2.6, we have for any $x \in P$ and $t \in[0,1]$,

$$
\begin{aligned}
& \left(L_{\tau} x\right)(t) \geq \begin{cases}\left(L_{\tau} x\right)(\eta) \frac{p_{0, \tau}(t)}{p_{0, \tau}(\eta)}, & t \in[0, \eta], \\
\left(L_{\tau} x\right)(\eta) \frac{q_{1, \tau}(t)+\gamma p_{\eta, \tau}(t)}{q_{1, \tau}(\eta)}, & t \in[\eta, 1],\end{cases} \\
& \geq \begin{cases}\left(L_{\tau} x\right)(\eta) \frac{t}{p_{0, \tau}(\eta)}, & t \in[0, \eta] \\
\left(L_{\tau} x\right)(\eta) \frac{1-t+\gamma(t-\eta)}{q_{1, \tau}(\eta)}, & t \in[\eta, 1]\end{cases} \\
& \geq\left(L_{\tau} x\right)(\eta) \frac{\gamma(1-\eta) t}{p_{0, \tau}(\eta)+q_{1, \tau}(\eta)} \\
& \left(L_{\tau} x\right)(\eta)=\frac{p_{0, \tau}(1)}{p_{0, \tau}(1)-\gamma p_{0, \tau}(\eta)}\left(\int_{0}^{\eta} q_{1, \tau}(\eta) \frac{p_{0, \tau}(s)}{p_{0, \tau}(1)} a(s) x(s) d s\right. \\
& \left.+\int_{\eta}^{1} p_{0, \tau}(\eta) \frac{q_{1, \tau}(s)}{q_{1, \tau}(0)} a(s) x(s) d s\right) \\
& \geq \frac{q_{1, \tau}(\eta)}{p_{0, \tau}(1)-\gamma p_{0, \tau}(\eta)} \int_{0}^{\eta} p_{0, \tau}(s) a(s) x(s) d s, \\
& \left(L_{\tau} x\right)(\eta)=\frac{p_{0, \tau}(1)}{p_{0, \tau}(1)-\gamma p_{0, \tau}(\eta)}\left(\int_{0}^{\eta} q_{1, \tau}(\eta) \frac{p_{0, \tau}(s)}{p_{0, \tau}(1)} a(s) x(s) d s\right. \\
& \left.+\int_{\eta}^{1} p_{0, \tau}(\eta) \frac{q_{1, \tau}(s)}{q_{1, \tau}(0)} a(s) x(s) d s\right) \\
& \geq \frac{p_{0, \tau}(\eta)}{p_{0, \tau}(1)-\gamma p_{0, \tau}(\eta)} \int_{\eta}^{1} q_{1, \tau}(s) a(s) x(s) d s .
\end{aligned}
$$


By (2.18) and Lemma 2.6, we have for any $t \in[0, \eta]$,

$$
\begin{aligned}
\left(L_{\tau} x\right)(t)= & \int_{0}^{t} q_{\eta, \tau}(t) \frac{p_{0, \tau}(s)}{p_{0, \tau}(\eta)} a(s) x(s) d s \\
& +\int_{t}^{\eta} p_{0, \tau}(t) \frac{q_{\eta, \tau}(s)}{q_{\eta, \tau}(0)} a(s) x(s) d s+\left(L_{\tau} x\right)(\eta) \frac{p_{0, \tau}(t)}{p_{0, \tau}(\eta)} \\
\leq & \int_{0}^{t} q_{\eta, \tau}(0) \frac{p_{0, \tau}(s)}{p_{0, \tau}(\eta)} a(s) x(s) d s+\int_{t}^{\eta} p_{0, \tau}(s) \frac{q_{\eta, \tau}(0)}{q_{\eta, \tau}(0)} a(s) x(s) d s+\left(L_{\tau} x\right)(\eta) \\
= & \int_{0}^{\eta} p_{0, \tau}(s) a(s) x(s) d s+\left(L_{\tau} x\right)(\eta) \\
\leq & \frac{q_{1, \tau}(0)+q_{1, \tau}(\eta)}{q_{1, \tau}(\eta)}\left(L_{\tau} x\right)(\eta)
\end{aligned}
$$

here we have used the facts that $q_{\eta, \tau}(0)=p_{0, \tau}(\eta)$ and $p_{0, \tau}(1)=q_{1, \tau}(0)$. From (2.19) and Lemma 2.6, we have for any $t \in[\eta, 1]$,

$$
\begin{aligned}
\left(L_{\tau} x\right)( & t) \\
\leq & \int_{\eta}^{t} q_{1, \tau}(s) \frac{p_{\eta, \tau}(1)}{p_{\eta, \tau}(1)} a(s) x(s) d s \\
& \quad+\int_{t}^{1} p_{\eta, \tau}(1) \frac{q_{1, \tau}(s)}{q_{1, \tau}(\eta)} a(s) x(s) d s+\left(L_{\tau} x\right)(\eta) \frac{q_{1, \tau}(t)+\gamma p_{\eta, \tau}(t)}{q_{1, \tau}(\eta)} \\
\leq & \int_{\eta}^{1} q_{1, \tau}(s) a(s) x(s) d s+\left(L_{\tau} x\right)(\eta) \frac{q_{1, \tau}(\eta)((1-t) /(1-\eta))+\gamma p_{\eta, \tau}(1)((t-\eta) /(1-\eta))}{q_{1, \tau}(\eta)} \\
\leq & \int_{\eta}^{1} q_{1, \tau}(s) a(s) x(s) d s+\left(L_{\tau} x\right)(\eta) \\
\leq & \frac{p_{0, \tau}(1)+p_{0, \tau}(\eta)}{p_{0, \tau}(\eta)}\left(L_{\tau} x\right)(\eta) ;
\end{aligned}
$$

here we have used the fact $p_{\eta, \tau}(1)=q_{1, \tau}(\eta)$. By (2.20) and (2.21), we have

$$
\left(L_{\tau}\right)(\eta) \geq \min \left\{\frac{q_{1, \tau}(\eta)}{q_{1, \tau}(0)+q_{1, \tau}(\eta)}, \frac{p_{0, \tau}(\eta)}{p_{0, \tau}(1)+p_{0, \tau}(\eta)}\right\}\left\|L_{\tau} x\right\| .
$$

By (2.17) and (2.22), we have

$$
\left(L_{\tau} x\right)(t) \geq \theta_{\tau}\left\|L_{\tau} x\right\| t
$$

This implies that $L_{\tau}: P \mapsto Q_{\tau}$.

Now we will show that $L_{\tau}: P \mapsto Q_{\tau}$ is completely continuous. It is easy to show that $L_{\tau}: P \mapsto Q_{\tau}$ is continuous and bounded. Let $B \subset P$ be a bounded set such that $\|x\| \leq R_{0}$ 
and $\left\|L_{\tau} x\right\| \leq R_{0}$ for some $R_{0}>0$. For any $\varepsilon>0$, by $\left(\mathrm{H}_{1}\right)$ there exists $\delta_{1}>0$ such that

$$
\begin{aligned}
& 2 R_{0} \int_{0}^{\delta_{1}} G_{[0, \eta]}^{(\tau)}(s, s) a(s) d s+2 R_{0} \int_{\eta-\delta_{1}}^{\eta} G_{[0, \eta]}^{(\tau)}(s, s) a(s) d s \\
& \quad \leq 2 R_{0} q_{\eta, \tau}(0) \int_{0}^{\delta_{1}} \frac{(\eta-s) s}{\eta^{2}} a(s) d s+2 R_{0} p_{0, \tau}(\eta) \int_{\eta-\delta_{1}}^{\eta} \frac{(\eta-s) s}{\eta^{2}} a(s) d s<\frac{\varepsilon}{3} .
\end{aligned}
$$

It is easy to see that there exists $\delta>0$ such that for any $t_{1}, t_{2} \in[0, \eta],\left|t_{1}-t_{2}\right|<\delta$,

$$
\begin{gathered}
R_{0} \int_{\delta_{1}}^{\eta-\delta_{1}}\left|G_{[0, \eta]}^{(\tau)}\left(t_{1}, s\right)-G_{[0, \eta]}^{(\tau)}\left(t_{2}, s\right)\right| a(s) d s<\frac{\varepsilon}{3}, \\
R_{0} \frac{\left|p_{0, \tau}\left(t_{2}\right)-p_{0, \tau}\left(t_{1}\right)\right|}{p_{0, \tau}(\eta)}<\frac{\varepsilon}{3} .
\end{gathered}
$$

By (2.24)-(2.25), we have for any $x \in B$ and $t_{1}, t_{2} \in[0, \eta],\left|t_{1}-t_{2}\right|<\delta$,

$$
\begin{aligned}
\left|\left(L_{\tau} x\right)\left(t_{2}\right)-\left(L_{\tau} x\right)\left(t_{1}\right)\right| \leq & \int_{0}^{\eta}\left|G_{[0, \eta]}^{(\tau)}\left(t_{2}, s\right)-G_{[0, \eta]}^{(\tau)}\left(t_{1}, s\right)\right| a(s) x(s) d s \\
& +\left(L_{\tau} x\right)(\eta) \frac{\left|p_{0, \tau}\left(t_{2}\right)-p_{0, \tau}\left(t_{1}\right)\right|}{p_{0, \tau}(\eta)} \\
\leq & 2 R_{0} \int_{0}^{\delta_{1}} G_{[0, \eta]}^{(\tau)}(s, s) a(s) d s \\
& +2 R_{0} \int_{\eta-\delta_{1}}^{\eta} G_{[0, \eta]}^{(\tau)}(s, s) a(s) d s \\
& +R_{0} \int_{\delta_{1}}^{\eta-\delta_{1}}\left|G_{[0, \eta]}^{(\tau)}\left(t_{1}, s\right)-G_{[0, \eta]}^{(\tau)}\left(t_{2}, s\right)\right| a(s) d s \\
& +R_{0} \frac{\left|p_{0, \tau}\left(t_{2}\right)-p_{0, \tau}\left(t_{1}\right)\right|}{p_{0, \tau}(\eta)}<\varepsilon .
\end{aligned}
$$

Thus, $L_{\tau}(B)$ is equicontinuous on $[0, \eta]$. Similarly, $L_{\tau}(B)$ is also equicontinuous on $[\eta, 1]$. By the Arzela-Ascoli theorem, $L_{\tau}(B) \subset C[0,1]$ is a relatively compact set. Therefore, $L_{\tau}$ : $P \mapsto Q_{\tau}$ is a completely continuous operator.

Finally, we show that $L_{\tau}: P \mapsto Q_{\tau}$ is increasing. For any $x_{1}, x_{2} \in P, x_{1} \leq x_{2} \in P$, let $y_{1}=L_{\tau} x_{1}$ and $y_{2}=L_{\tau} x_{2}, u=y_{2}-y_{1}$. Then, by Lemma 2.7, we have

$$
\begin{gathered}
-u^{\prime \prime}(t)+\tau a(t) u(t)=a(t)\left(x_{2}(t)-x_{1}(t)\right) \geq 0, \quad t \in(0,1), \\
u(0)=0=u(1)-\gamma u(\eta) .
\end{gathered}
$$

Then Lemma 2.9 implies that $u(t) \geq 0$ for $t \in[0,1]$, and so, $y_{2} \geq y_{1}$. The proof is complete.

Lemma 2.11. Assume $\left(H_{1}\right)$ and $\left(H_{2}\right)$ hold. Let $\lambda>0$ be fixed. If there exists $R_{\lambda}>0$ such that $\left(2.1_{n}^{\lambda}\right)$ has at least one positive solution $x_{n}$ with $\left\|x_{n}\right\| \leq R_{\lambda}$ for each positive integer $n$, then there exist $\bar{x} \in C[0,1]$ and a subsequence $\left\{x_{n_{k}}\right\}_{k=1}^{+\infty}$ of $\left\{x_{n}\right\}_{n=1}^{+\infty}$ such that $x_{n_{k}} \rightarrow \bar{x}$ as $k \rightarrow+\infty$. Moreover, $\bar{x}$ is a positive solution of the BVP $\left(1.1_{\lambda}\right)$ 
Proof. Let $z_{0}(t)=1$ for $t \in[0,1]$, and $z_{\lambda}(t)=\lambda g\left(R_{\lambda}+1\right)\left(L_{\tau} z_{0}\right)(t)$ for $t \in[0,1]$. Since $L_{0}$ is increasing and $g$ is nonincreasing, then we have for any $n \in \mathbb{N}$,

$$
x_{n}(t)=\lambda\left(L_{0} F_{n} x_{n}\right)(t) \geq \lambda g\left(R_{\lambda}+1\right)\left(L_{0} z_{0}\right)(t)=z_{\lambda}(t), \quad t \in[0,1] .
$$

Let us define the function $F$ by

$$
F(t)=\int_{t}^{1}(1-s) a(s) d s, \quad t \in(0,1]
$$

Obviously, $F \in C(0,1], F(1)=0$, and $F$ is nonincreasing on $(0,1]$. For each $n \in \mathbb{N}, x_{n}$ is a concave function on $[0,1]$. Then there exists $t^{n} \in(0,1)$ such that $x_{n}^{\prime}\left(t^{n}\right)=0$. By $\left(\mathrm{H}_{2}\right)$, we have

$$
-x_{n}^{\prime \prime}(t) \leq \lambda a(t) g\left(x_{n}(t)\right)\left(1+\frac{\bar{h}\left(R_{\lambda}\right)}{g\left(R_{\lambda}+1\right)}\right), \quad t \in(0,1)
$$

where $\bar{h}\left(R_{\lambda}\right)=\max _{s \in\left[0, R_{\lambda}\right]} h(s)$. Integrate $(2.30)$ from $t^{n}$ to $t\left(t \in\left(t^{n}, 1\right)\right)$ to obtain

$$
\frac{-x_{n}^{\prime}(t)}{g\left(x_{n}(t)\right)} \leq \lambda\left(1+\frac{\bar{h}\left(R_{\lambda}\right)}{g\left(R_{\lambda}+1\right)}\right) \int_{t^{n}}^{t} a(s) d s .
$$

Then integrate (2.31) from $t^{n}$ to 1 to obtain

$$
\int_{x_{n}(1)}^{x_{n}\left(t^{n}\right)} \frac{d s}{g(s)} \leq \lambda\left(1+\frac{\bar{h}\left(R_{\lambda}\right)}{g\left(R_{\lambda}+1\right)}\right) \int_{t^{n}}^{1}(1-s) a(s) d s=\lambda\left(1+\frac{\bar{h}\left(R_{\lambda}\right)}{g\left(R_{\lambda}+1\right)}\right) F\left(t^{n}\right) .
$$

On the other hand, by (2.28), we have

$$
\int_{x_{n}(1)}^{x_{n}\left(t^{n}\right)} \frac{d s}{g(s)} \geq \frac{x_{n}\left(t^{n}\right)-x_{n}(1)}{g\left(x_{n}(1)\right)} \geq \frac{x_{n}(\eta)(1-\gamma)}{g\left(x_{n}(1)\right)} \geq \frac{z_{\lambda}(\eta)(1-\gamma)}{g\left(z_{\lambda}(1)\right)} .
$$

By (2.32) and (2.33), we have

$$
F\left(t^{n}\right) \geq\left[\lambda\left(1+\frac{\bar{h}\left(R_{\lambda}\right)}{g\left(R_{\lambda}+1\right)}\right)\right]^{-1} \frac{z_{\lambda}(\eta)(1-\gamma)}{g\left(z_{\lambda}(1)\right)} .
$$

Let $\beta_{0} \in(0,1]$ be such that

$$
F\left(\beta_{0}\right)=\left[\lambda\left(1+\frac{\bar{h}\left(R_{\lambda}\right)}{g\left(R_{\lambda}+1\right)}\right)\right]^{-1} \frac{z_{\lambda}(\eta)(1-\gamma)}{g\left(z_{\lambda}(1)\right)}
$$

Then (2.34) implies that $t^{n} \leq \beta_{0}$. Similarly, we can show that there exists $\alpha_{0}>0$ such that $t^{n} \geq \alpha_{0}$ for each $n \in \mathbb{N}$. Let us define the function $I: \mathbb{R}^{+} \mapsto \mathbb{R}^{+}$by $I(x)=\int_{0}^{x} d s / g(s)$ for 
$x \in \mathbb{R}^{+}$. For any $t_{1}, t_{2} \in\left[\beta_{0}, 1\right], t_{1}<t_{2}$, by $(2.31)$, we have

$$
\begin{aligned}
I\left(x_{n}\left(t_{1}\right)\right)-I\left(x_{n}\left(t_{2}\right)\right) & =\int_{x_{n}\left(t_{2}\right)}^{x_{n}\left(t_{1}\right)} \frac{d s}{g(s)}=\int_{t_{1}}^{t_{2}}-\frac{x_{n}^{\prime}(s) d s}{g\left(x_{n}(s)\right)} \\
& \leq \lambda\left(1+\frac{\bar{h}\left(R_{\lambda}\right)}{g\left(R_{\lambda}+1\right)}\right) \int_{t_{1}}^{t_{2}} d t \int_{0}^{t} a(s) d s \\
& \leq \lambda\left(1+\frac{\bar{h}\left(R_{\lambda}\right)}{g\left(R_{\lambda}+1\right)}\right)\left(\int_{t_{1}}^{t_{2}}\left(t_{2}-s\right) a(s) d s+\left(t_{2}-t_{1}\right) \int_{0}^{t_{1}} a(s) d s\right) \\
& \leq \lambda\left(1+\frac{\bar{h}\left(R_{\lambda}\right)}{g\left(R_{\lambda}+1\right)}\right)\left(\int_{t_{1}}^{t_{2}}(1-s) a(s) d s+\left(t_{2}-t_{1}\right) \int_{0}^{1-\left(t_{2}-t_{1}\right)} a(s) d s\right) .
\end{aligned}
$$

This and the inequalities (2.21) in [11] imply that the set $I\left(\left\{x_{n}\right\}_{n=1}^{+\infty}\right)$ is equicontinuous on $\left[\beta_{0}, 1\right]$. It is easy to see that $I^{-1}$, the inverse function of $I$, is uniformly continuous on $\left[0, I\left(R_{\lambda}\right)\right]$. Therefore, the set $\left\{x_{n}\right\}_{n=1}^{+\infty}$ is equcontinuous on $\left[\beta_{0}, 1\right]$. Similarly, $\left\{x_{n}\right\}_{n=1}^{+\infty}$ is equcontinuous on $\left[0, \alpha_{0}\right]$.

From (2.30), we have for any $t \in\left[\alpha_{0}, \beta_{0}\right]$,

$$
\left|x_{n}^{\prime}(t)\right| \leq \lambda\left(g\left(\min _{t \in\left[\alpha_{0}, \beta_{0}\right]} z_{\lambda}(t)\right)+\bar{h}\left(R_{\lambda}\right)\right) \int_{\alpha_{0}}^{\beta_{0}} a(s) d s .
$$

Thus, $\left\{x_{n}\right\}_{n=1}^{+\infty}$ is equcontinuous on $\left[\alpha_{0}, \beta_{0}\right]$. Then, by the Arzela-Ascoli theorem, we see that $\left\{x_{n}\right\}_{n=1}^{+\infty} \subset C[0,1]$ is a relatively compact set. Thus, there exist $\bar{x} \in C[0,1]$ and a subsequence $\left\{x_{n_{k}}\right\}_{k=1}^{+\infty}$ of $\left\{x_{n}\right\}_{n=1}^{+\infty}$ such that $x_{n_{k}} \rightarrow \bar{x}$. By a standard argument (see [11]), we have that $\bar{x}$ is a positive solution of the BVP $\left(1.1_{\lambda}\right)$. The proof is complete.

Lemma 2.12. Assume that $\left(H_{1}\right)$ and $\left(H_{2}\right)$ hold. Then for small enough $\lambda>0$, the $B V P\left(1.1_{\lambda}\right)$ has at least one positive solution.

Proof. Let $R_{0}>0$ and $\lambda_{0}$ be such that

$$
0<\lambda_{0}<\frac{1}{2} \int_{\gamma R_{0}}^{R_{0}} \frac{d s}{g(s)}\left(\int_{0}^{1} s(1-s) a(s) d s\right)^{-1}\left(1+\frac{\bar{h}\left(R_{0}\right)}{g\left(R_{0}+1\right)}\right)^{-1}
$$

By Lemma 2.10, $\lambda_{0} L_{0} F_{n}: P \mapsto Q_{0}$ is a completely continuous operator for each $n \in \mathbb{N}$. Now we will show that

$$
\mu \lambda_{0} L_{0} F_{n} u \neq u, \quad \mu \in[0,1], u \in \partial B\left(\theta, R_{0}\right), n \in \mathbb{N},
$$

where $B\left(\theta, R_{0}\right)=\left\{x \in Q_{0} \mid\|x\|<R_{0}\right\}$. Suppose (2.39) is not true. Then there exist $\mu_{0} \in$ $[0,1], u_{0} \in \partial B\left(\theta, R_{0}\right)$, and $n_{0} \in \mathbb{N}$ such that $\mu_{0} \lambda_{0} L_{0} F_{n_{0}} u_{0}=u_{0}$. Obviously, $\mu_{0}>0$. 
By Lemma 2.7, we have

$$
\begin{gathered}
u_{0}^{\prime \prime}(t)+\mu_{0} \lambda_{0} a(t)\left(g\left(u_{0}+\frac{1}{n_{0}}\right)+h\left(u_{0}\right)\right)=0, \quad 0<t<1, \\
u_{0}(0)=0=u_{0}(1)-\gamma u_{0}(\eta) .
\end{gathered}
$$

Thus $u_{0}$ is a concave function on $[0,1]$, and there exists $t_{0} \in(0,1)$ such that $u_{0}^{\prime}\left(t_{0}\right)=0$.

A similar argument as in the proof of (2.32) guarantees that

$$
\begin{aligned}
\int_{u_{0}(1)}^{u_{0}\left(t_{0}\right)} \frac{d s}{g(s)} & \leq \lambda_{0} \mu_{0}\left(1+\frac{\bar{h}\left(R_{0}\right)}{g\left(R_{0}+1\right)}\right) \int_{t_{0}}^{1}(1-s) a(s) d s \\
& \leq \frac{\lambda_{0} \mu_{0}}{t_{0}}\left(1+\frac{\bar{h}\left(R_{0}\right)}{g\left(R_{0}+1\right)}\right) \int_{0}^{1} s(1-s) a(s) d s, \\
\int_{u_{0}(0)}^{u_{0}\left(t_{0}\right)} \frac{d s}{g(s)} & \leq \frac{\lambda_{0} \mu_{0}}{1-t_{0}}\left(1+\frac{\bar{h}\left(R_{0}\right)}{g\left(R_{0}+1\right)}\right) \int_{0}^{1} s(1-s) a(s) d s .
\end{aligned}
$$

Since $u_{0}\left(t_{0}\right)=R_{0}$ and $u_{0}(1)=\gamma u_{0}(\eta) \leq \gamma R_{0}$, by (2.41), we have

$$
\lambda_{0} \geq \frac{1}{2}\left(\left(1+\frac{\bar{h}\left(R_{0}\right)}{g\left(R_{0}+1\right)}\right) \int_{0}^{1} s(1-s) a(s) d s\right)^{-1} \int_{\gamma R_{0}}^{R_{0}} \frac{d s}{g(s)},
$$

which contradicts (2.38). Therefore, (2.39) holds, and so

$$
i\left(\lambda_{0} L_{0} F_{n}, B\left(\theta, R_{0}\right), Q_{0}\right)=1, \quad n \in \mathbb{N} .
$$

This means that for each $n \in \mathbb{N}$, the operator $\lambda_{0} L_{0} F_{n}$ has at least one positive fixed point $x_{n}$ such that $\left\|x_{n}\right\| \leq R_{0}$. By Lemma 2.7, the BVP $\left(2.1_{n}^{\lambda}\right)$ has a positive solution $x_{n}$ such that $\left\|x_{n}\right\| \leq R_{0}$. Then by Lemma 2.11 , the BVP $\left(1.1_{\lambda}\right)$ has at least one positive solution. The proof is complete.

LEMma 2.13. Let $\alpha(t)$ and $\beta(t)$ be lower and upper solutions of $\left(2.1_{n}^{\lambda}\right)$ for some $n \in \mathbb{N}$ and $\lambda>0,0 \leq \alpha(t) \leq \beta(t)$. Then $\left(2.1_{n}^{\lambda}\right)$ has at least one positive solution $u_{n, \lambda}$ such that

$$
\alpha(t) \leq u_{n, \lambda}(t) \leq \beta(t), \quad t \in[0,1] .
$$

Proof. Let us define the function $F_{n}^{*}$ by

$$
\left(F_{n}^{*} x\right)(t)= \begin{cases}g\left(\beta(t)+\frac{1}{n}\right)+h(\beta(t)), & x \geq \beta(t), \\ g\left(x+\frac{1}{n}\right)+h(x), & \alpha(t)<x<\beta(t), \\ g\left(\alpha(t)+\frac{1}{n}\right)+h(\alpha(t)), & \alpha(t)<x,\end{cases}
$$


for $x \in P$. Then there exists a constant $C_{n}>0$ such that $0 \leq\left(F_{n}^{*} x\right)(t) \leq C_{n}$ for $x \in P$. Now Lemma 2.10 and Schauder's fixed point theorem guarantees that the operator $\lambda L_{0} F_{n}^{*}$ has at least one fixed point. Then the BVP

$$
\begin{gathered}
u^{\prime \prime}(t)+\lambda a(t)\left(F_{n}^{*} u\right)(t)=0, \quad t \in(0,1), \\
u(0)=0=u(1)-\gamma u(\eta)
\end{gathered}
$$

has at least one solution $u_{n, \lambda}(t)$. Now, we will show that $\alpha(t) \leq u_{n, \lambda}(t) \leq \beta(t)$ for $t \in$ $[0,1]$. Suppose that $\varepsilon_{0}=\max _{t \in[0,1]}\left\{u_{n, \lambda}(t)-\beta(t)\right\}>0$. Let $y_{n, \lambda}(t)=u_{n, \lambda}(t)-\beta(t)$. Then, $y_{n, \lambda}(t) \leq \varepsilon_{0}$ for $t \in[0,1]$. Let $t_{0} \in\left[t_{1}, t_{2}\right] \subset[0,1]$ be such that

(a) $y_{n, \lambda}\left(t_{0}\right)=\varepsilon_{0}$

(b) $y_{n, \lambda}(t)>0$ for $t \in\left(t_{1}, t_{2}\right)$,

(c) $\left[t_{1}, t_{2}\right]$ is the maximal interval which has the properties (a) and (b).

Then we have the following three cases.

(1) If $t_{0} \in(0,1)$, then $t_{0} \in\left(t_{1}, t_{2}\right), y_{n, \lambda}^{\prime}\left(t_{0}\right)=0$. Also

$$
-y_{n, \lambda}^{\prime \prime}(t) \leq \lambda a(t)\left[g\left(\beta(t)+\frac{1}{n}\right)+h(\beta(t))-g\left(\beta(t)+\frac{1}{n}\right)-h(\beta(t))\right]=0
$$

for $t \in\left[t_{1}, t_{2}\right]$. Then $y_{n, \lambda}^{\prime}(t) \leq 0$ for $t \in\left(t_{1}, t_{0}\right)$, and $y_{n, \lambda}^{\prime}(t) \geq 0$ for $t \in\left(t_{0}, t_{2}\right)$. Since $y_{n, \lambda}\left(t_{0}\right)=\max _{t \in[0,1]} y_{n, \lambda}(t)$, then $y_{n, \lambda}(t)=\varepsilon_{0}$ for $t \in\left[t_{1}, t_{2}\right]$, contradicting the properties (b) and (c).

(2) If $t_{0}=1$, then $y_{n, \lambda}(1)=u_{n, \lambda}(1)-\beta(1) \leq \gamma\left(u_{n, \lambda}(\eta)-\beta(\eta)\right)=\gamma y_{n, \lambda}(\eta) \leq \gamma y_{n, \lambda}(1)$, and so $y_{n, \lambda}(1)=0$, a contradiction.

(3) If $t_{0}=0$, then $y_{n, \lambda}(0)=u_{n, \lambda}(0)-\beta(0)<0$, a contradiction.

Therefore, $\beta(t) \geq u_{n, \lambda}(t)$ for $t \in[0,1]$. Similarly, we can show that $\alpha(t) \leq u_{n, \lambda}(t)$ for $t \in[0,1]$. Thus, $u_{n, \lambda}(t)$ is a positive solution of $\left(2.1_{n}^{\lambda}\right)$. The proof is complete.

\section{Proof of the main results}

Proof of Theorem 2.1. Let

$$
\Lambda=\left\{\lambda \in(0,+\infty) \mid\left(1.1_{\lambda}\right) \text { has at least one positive solution }\right\}
$$

By Lemma 2.12, $\Lambda \neq \varnothing$. Assume that $\lambda_{0} \in \Lambda$. Then we can show that

(1) $\lambda^{\prime} \in \Lambda$ for any $0<\lambda^{\prime} \leq \lambda_{0}$,

(2)

$$
\lambda_{0} \leq \frac{p_{0,0}(1)-\gamma p_{0,0}(\eta)}{q_{1,0}(\eta)}\left(\int_{(1 / 2) \eta}^{\eta} s^{2} a(s) d s\right)^{-1} \max \left\{\frac{1}{b_{0} \theta_{0}^{\omega}}, \frac{1}{g(2)}\right\}
$$

Assume that $\left(1.1_{\lambda}\right)$ has a positive solution $z_{0}(t)$. It is easy to see that $z_{0}(t)$ and 0 are upper and lower solutions of $\left(2.1_{\lambda^{\prime}}^{n}\right)$ for each $n \in \mathbb{N}$, respectively. By Lemma 2.13, for each $n \in \mathbb{N}$, $\left(2.1_{\lambda^{\prime}}^{n}\right)$ has a positive solution $x_{n, \lambda^{\prime}}$ such that $0 \leq x_{n, \lambda^{\prime}} \leq z_{0}$. Thus, by Lemma 2.11, there exist $\bar{x}_{\lambda^{\prime}} \in C[0,1]$ and a subsequence $\left\{x_{n_{k}, \lambda^{\prime}}\right\}_{k=1}^{+\infty}$ of $\left\{x_{n, \lambda^{\prime}}\right\}_{n=1}^{+\infty}$ such that $x_{n_{k}, \lambda^{\prime}} \rightarrow \bar{x}_{\lambda^{\prime}}$ as $k \rightarrow+\infty$ and $\bar{x}_{\lambda^{\prime}}$ is a positive solution of $\left(1.1_{\lambda^{\prime}}\right)$. Thus, $\lambda^{\prime} \in \Lambda$. 
From Lemma 2.7, we have $x_{n_{k}, \lambda^{\prime}}=\lambda^{\prime} L_{0} F_{n_{k}} x_{n_{k}, \lambda^{\prime}}$. Then by Lemma 2.10,

$$
x_{n_{k}, \lambda^{\prime}}(t) \geq \theta_{0}\left\|x_{n_{k}, \lambda^{\prime}}\right\| t, \quad t \in[0,1] .
$$

If $\left\|x_{n_{k}, \lambda^{\prime}}\right\| \leq 1$, then by $\left(\mathrm{H}_{2}\right)$, we have

$$
\begin{aligned}
1 & \geq\left\|x_{n_{k}, \lambda^{\prime}}\right\| \geq x_{n_{k}, \lambda^{\prime}}(\eta) \geq \frac{g(2) \lambda^{\prime} p_{0,0}(1)}{p_{0,0}-\gamma p_{0,0}(\eta)} \int_{0}^{1} G_{[0,1]}^{(0)}(\eta, s) a(s) d s \\
& =\frac{g(2) \lambda^{\prime} p_{0,0}(1)}{p_{0,0}(1)-\gamma p_{0,0}(\eta)}\left[\int_{0}^{\eta} q_{1,0}(\eta) \frac{p_{0,0}(s)}{p_{0,0}(1)} a(s) d s+\int_{\eta}^{1} p_{0,0}(\eta) \frac{q_{1,0}(s)}{q_{1,0}(0)} a(s) d s\right] \\
& \geq \frac{g(2) \lambda^{\prime} q_{1,0}(\eta)}{p_{0,0}(1)-\gamma p_{0,0}(\eta)} \int_{(1 / 2) \eta}^{\eta} s a(s) d s,
\end{aligned}
$$

and so

$$
\lambda^{\prime} \leq \frac{p_{0,0}(1)-\gamma p_{0,0}(\eta)}{g(2) q_{1,0}(\eta)}\left(\int_{(1 / 2) \eta}^{\eta} s a(s) d s\right)^{-1} .
$$

If $\left\|x_{n_{k}, \lambda^{\prime}}\right\| \geq 1$, then by $\left(\mathrm{H}_{2}\right)$ and (3.3), we have

$$
\begin{aligned}
\left\|x_{n_{k}, \lambda^{\prime}}\right\| & \geq x_{n_{k}, \lambda^{\prime}}(\eta) \\
& \geq \frac{b_{0} \lambda^{\prime} p_{0,0}(1)}{p_{0,0}(1)-\gamma p_{0,0}(\eta)} \int_{0}^{1} G_{[0,1]}^{(0)}(\eta, s) a(s)\left[x_{n_{k}, \lambda^{\prime}}\right]^{w} d s \\
& \geq \frac{b_{0} \lambda^{\prime} q_{1,0}(\eta) \theta_{0}^{\omega}}{p_{0,0}(1)-\gamma p_{0,0}(\eta)} \int_{(1 / 2) \eta}^{\eta} s^{2} a(s) d s\left\|x_{n_{k}, \lambda^{\prime}}\right\|^{w} \\
& \geq \frac{b_{0} \lambda^{\prime} q_{1,0}(\eta) \theta_{0}^{\omega}}{p_{0,0}(1)-\gamma p_{0,0}(\eta)} \int_{(1 / 2) \eta}^{\eta} s^{2} a(s) d s\left\|x_{n_{k}, \lambda^{\prime}}\right\|,
\end{aligned}
$$

and so

$$
\lambda^{\prime} \leq \frac{p_{0,0}(1)-\gamma p_{0,0}(\eta)}{b_{0} \theta_{0}^{\omega} q_{1,0}(\eta)}\left(\int_{(1 / 2) \eta}^{\eta} s^{2} a(s) d s\right)^{-1} .
$$

Then, (3.2) follows from (3.5) and (3.7), and (3.2) implies that $\Lambda$ is a bounded set. Let $\lambda^{*}=\sup \Lambda$. Therefore, $\left(1.1_{\lambda}\right)$ has at least one positive solution for $0<\lambda<\lambda^{*}$.

Finally, we will show that $\lambda^{*} \in \Lambda$ if $\omega>1$. Let $\left\{\lambda_{n}\right\} \subset \Lambda$ be an increasing number sequence such that $\lambda_{n} \rightarrow \lambda^{*}$ as $n \rightarrow+\infty$, and $\lambda_{n} \geq \lambda^{*} / 2$ for $n=1,2, \ldots$. Assume that $\left(1.1_{\lambda_{n}}\right)$ has positive solution $z_{n}$ for each $n \in \mathbb{N}$. Then $z_{n}$ is an upper solution of $\left(2.1_{\lambda_{n}}^{k}\right)$ and 0 is a lower solution of $\left(2.1_{\lambda_{n}}^{k}\right)$ for each $k \in \mathbb{N}$. By Lemma $2.13,\left(2.1_{\lambda_{n}}^{k}\right)$ has a positive solution $z_{n, k}$ such that $0 \leq z_{n, k} \leq z_{n}$. Then, by Lemma 2.7,

$$
z_{n, k}=\lambda_{n} L_{0} F_{k} z_{n, k}
$$


Let $k \in \mathbb{N}$ be fixed. Now we will show that $\left\{z_{n, k}\right\}_{n=1}^{+\infty}$ is bounded. In fact, by (3.8) and Lemmas 2.6 and 2.10, we have

$$
\begin{aligned}
\left\|z_{n, k}\right\| & \geq\left(\lambda_{n} L_{0} F_{k} z_{n, k}\right)(\eta) \\
& \geq \frac{\lambda^{*} p_{0,0}(1)}{2\left(p_{0,0}(1)-\gamma p_{0,0}(\eta)\right)} \int_{(1 / 2) \eta}^{\eta} q_{1,0}(\eta) \frac{p_{0,0}(s)}{p_{0,0}(1)} a(s) h\left(z_{n, k}(s)\right) d s \\
& \geq \frac{\lambda^{*} b_{0} q_{1,0}(\eta)}{2\left(p_{0,0}(1)-\gamma p_{0,0}(\eta)\right)} \int_{(1 / 2) \eta}^{\eta} p_{0,0}(s) a(s)\left[z_{n, k}(s)\right]^{w} d s \\
& \geq \frac{\lambda^{*} b_{0} \theta_{0}^{w} q_{1,0}(\eta)}{2\left(p_{0,0}(1)-\gamma p_{0,0}(\eta)\right)} \int_{(1 / 2) \eta}^{\eta} s^{2} a(s)\left\|z_{n, k}(s)\right\|^{w} d s,
\end{aligned}
$$

and so

$$
\left\|z_{n, k}\right\| \leq\left[\frac{2\left(p_{0,0}(1)-\gamma p_{0,0}(\eta)\right)}{\lambda^{*} q_{1,0}(\eta) b_{0} \theta_{0}^{w}}\left(\int_{(1 / 2) \eta}^{\eta} s^{2} a(s) d s\right)^{-1}\right]^{1 /(w-1)} .
$$

This means that $\left\{z_{n, k}\right\}_{n=1}^{+\infty}$ is a bounded set. Using the fact that $L_{0}: P \mapsto Q_{0}$ is a completely continuous operator and $\left\{\lambda_{n}\right\}_{n=1}^{+\infty}$ is a bounded set, we see that $\left\{z_{n, k}\right\}$ is a relatively compact set. Without loss of generality, we assume that $z_{n, k} \rightarrow z_{0, k}$ as $n \rightarrow+\infty$. Now the Lebesgue dominant convergence theorem guarantees that $z_{0, k}=\lambda^{*} L_{0} F_{k} z_{0, k}$. Then, by Lemma $2.7, z_{0, k}$ is a positive solution of $\left(1.1_{\lambda^{*}}^{k}\right)$. By Lemma $2.11,\left(1.1_{\lambda^{*}}\right)$ has a positive solution $u^{*}$. The proof is complete.

Proof of Theorem 2.2. Let $\lambda^{*}$ be defined as in Theorem 2.1 and let $\lambda \in\left(0, \lambda^{*}\right)$ be fixed. Let us define the nonlinear operators $F$ and $T_{\lambda}$ by

$$
(F x)(t)=f(x(t))+M x(t), t \in[0,1], \quad x \in P,
$$

and $\left(T_{\lambda} x\right)(t)=\left(\lambda L_{\lambda M} F x\right)(t)$ for all $x \in P$ and $t \in[0,1]$. It follows from Lemma 2.7 that to show that $\left(1.1_{\lambda}\right)$ has at least two positive solutions, we only need to show that the operator $T_{\lambda}$ has at least two fixed points.

Let $z_{0}(t)=1$ for $t \in[0,1]$ and $\Omega_{\lambda}=\left\{x \in Q_{\lambda M} \mid \exists \tau>0\right.$ such that $\left.T_{\lambda} x \leq u^{*}-\tau\left(L_{\lambda M} z_{0}\right)(t)\right\}$. Since $u^{*}$ is a positive solution of $\left(1.1_{\lambda^{*}}\right)$, then

$$
\begin{gathered}
-\left(u^{*}\right)^{\prime \prime}(t)+\lambda M a(t) u^{*}(t)=\lambda a(t)\left(F u^{*}\right)(t)+\left(\lambda^{*}-\lambda\right) a(t) f\left(u^{*}(t)\right), \quad 0<t<1, \\
u^{*}(0)=0, \quad u^{*}(1)=\gamma u^{*}(\eta) .
\end{gathered}
$$

By Lemma 2.7, we have $u^{*}=T_{\lambda} u^{*}+\left(\lambda^{*}-\lambda\right) L_{\lambda M} f\left(u^{*}\right)$. Since $L_{\lambda M}$ is increasing and $f\left(u^{*}\right) \geq c$, then we have

$$
T_{\lambda} u^{*} \leq u^{*}-c\left(\lambda^{*}-\lambda\right)\left(L_{\lambda M} z_{0}\right)(t) .
$$

This means that $u^{*} \in \Omega_{\lambda}$, and so $\Omega_{\lambda} \neq \varnothing$. 
For any $x_{0} \in \Omega_{\lambda}$, by Lemma 2.10 , we have

$$
\begin{aligned}
\left\|u^{*}\right\| & \geq\left(T_{\lambda} x\right)(\eta) \geq \frac{\lambda p_{0, \lambda M}(1)}{p_{0, \lambda M}(1)-\gamma p_{0, \lambda M}(\eta)} \int_{(1 / 2) \eta}^{\eta} q_{1, \lambda M}(\eta) \frac{p_{0, \lambda M}(s)}{p_{0, \lambda M}(1)} a(s) h(x(s)) d s \\
& \geq \frac{\lambda q_{1, \lambda M}(\eta)}{p_{0, \lambda M}(1)-\gamma p_{0, \lambda M}(\eta)} \int_{(1 / 2) \eta}^{\eta} s a(s) b_{0}\left[x_{0}(s)\right]^{w} d s \\
& \geq \frac{b_{0} \lambda \theta_{\lambda M}^{\omega} q_{1, \lambda M}(\eta)}{p_{0, \lambda M}(1)-\gamma p_{0, \lambda M}(\eta)} \int_{(1 / 2) \eta}^{\eta} s^{2} a(s)\left\|x_{0}(s)\right\|^{w} d s,
\end{aligned}
$$

and so

$$
\left\|x_{0}\right\| \leq\left[\frac{p_{0, \lambda M}(1)-\gamma p_{0, \lambda M}(\eta)}{b_{0} \lambda \theta_{\lambda M}^{\omega} q_{1, \lambda M}(\eta)}\left(\int_{(1 / 2) \eta}^{\eta} s^{2} a(s) d s\right)^{-1}\left\|u^{*}\right\|\right]^{1 / w}=: R_{0} .
$$

This means that $\Omega_{\lambda}$ is a bounded set.

For any $x_{0} \in \Omega_{\lambda}$, there exists $\tau_{0}>0$ such that $T_{\lambda} x_{0} \leq u^{*}-\tau_{0}\left(L_{\lambda M} z_{0}\right)(t)$. For any $x \in$ $Q_{\lambda M}$, by Lemma 2.10, we have for $t \in[0,1]$,

$$
\left(T_{\lambda} x\right)(t)-\left(T_{\lambda} x_{0}\right)(t)=\left(\lambda L_{\lambda M}\left(F x-F x_{0}\right)\right)(t) \leq \lambda\left\|F x-F x_{0}\right\|\left(L_{\lambda M} z_{0}\right)(t),
$$

and since $F$ is continuous on $Q_{\lambda M}$, then there exists $\delta>0$ such that

$$
\lambda\left\|F x-F x_{0}\right\| \leq \frac{\tau_{0}}{2}
$$

for any $x \in Q_{\lambda M}$ with $\left\|x-x_{0}\right\|<\delta$.

By (3.16) and (3.17), we have

$$
\left(T_{\lambda} x\right)(t) \leq T_{\lambda} x_{0}(t)+\frac{\tau_{0}}{2}\left(L_{\lambda M} z_{0}\right)(t) \leq u^{*}(t)-\frac{\tau_{0}}{2}\left(L_{\lambda M} z_{0}\right)(t), \quad t \in[0,1],
$$

for any $x \in Q_{\lambda M}$ with $\left\|x-x_{0}\right\|<\delta$. This implies that $x \in \Omega_{\lambda}$, and so $\Omega_{\lambda}$ is an open set.

Now we will show that

$$
\mu T_{\lambda} x \neq x, \quad x \in \partial \Omega_{\lambda}, \mu \in[0,1] .
$$

Suppose (3.19) is not true. Then there exist $x_{0} \in \partial \Omega_{\lambda}, \mu_{0} \in[0,1]$ such that $\mu_{0} T_{\lambda} x_{0}=x_{0}$. Obviously, $T_{\lambda} x_{0} \leq u *$, and so $x_{0}=\mu_{0} T_{\lambda} x_{0} \leq u^{*}$. Since $T_{\lambda}$ is increasing, we have

$$
T_{\lambda} x_{0} \leq T_{\lambda} u^{*} \leq u^{*}-c\left(\lambda^{*}-\lambda\right)\left(L_{\lambda M} z_{0}\right)(t) .
$$

This implies that $x_{0} \in \Omega_{\lambda}$, a contradiction. Thus, (3.19) holds, and so

$$
i\left(T_{\lambda}, \Omega_{\lambda}, Q_{\lambda M}\right)=i\left(\theta, \Omega_{\lambda}, Q_{\lambda M}\right)=1 .
$$

Let

$$
R_{0}^{\prime}=\left[\frac{p_{0, \lambda M}(1)-\gamma p_{0, \lambda M}(\eta)}{b_{0} \theta_{M}^{w} \lambda q_{1, \lambda M}(\eta)}\left(\int_{(1 / 2) \eta}^{\eta} s^{2} a(s) d s\right)^{-1}\right]^{1 /(w-1)},
$$


and $R_{1}>\max \left\{R_{0}, R_{0}^{\prime}\right\}$. For any $x \in \partial\left(B\left(\theta, R_{1}\right) \cap Q_{\lambda M}\right)$, we have

$$
\begin{aligned}
\left\|T_{\lambda} x\right\| & \geq(\lambda T x)(\eta) \geq \frac{\lambda p_{0, \lambda M}(1)}{p_{0, \lambda M}(1)-\gamma p_{0, \lambda M}(\eta)} \int_{\eta / 2}^{\eta} q_{1, \lambda M}(\eta) \frac{p_{0, \lambda M}(s)}{p_{0, \lambda M}(1)} a(s) h(x(s)) d s \\
& \geq \frac{\lambda b_{0} q_{1, \lambda M}(\eta)}{p_{0, \lambda M}(1)-\gamma p_{0, \lambda M}(\eta)} \int_{\eta / 2}^{\eta} s a(s)[x(s)]^{w} d s \\
& \geq \frac{\theta_{M}^{w} \lambda b_{0} q_{1, \lambda M}(\eta)}{p_{0, \lambda M}(1)-\gamma p_{0, \lambda M}(\eta)} \int_{\eta / 2}^{\eta} s^{2} a(s)\|x(s)\|^{w} d s>R_{1} .
\end{aligned}
$$

Then, we have

$$
i\left(T_{\lambda}, B\left(\theta, R_{1}\right) \cap Q_{\lambda M}, Q_{\lambda M}\right)=0 .
$$

By (3.21) and (3.24), we have

$$
i\left(\lambda,\left(B\left(\theta, R_{1}\right) \cap Q_{\lambda M}\right) \backslash \bar{\Omega}_{\lambda}, Q_{\lambda M}\right)=0-1=-1 .
$$

It follows from (3.21) and (3.25) that $T_{\lambda}$ has at least two fixed points in $\left(B\left(\theta, R_{1}\right) \cap\right.$ $\left.Q_{\lambda M}\right) \backslash \bar{\Omega}_{\lambda}$ and $\Omega_{\lambda}$, respectively. Thus $\left(1.1_{\lambda}\right)$ has at least two positive solutions for $0<\lambda<$ $\lambda^{*}$.

\section{References}

[1] Y. S. Choi, A singular boundary value problem arising from near-ignition analysis of flame structure, Differential and Integral Equations. An International Journal for Theory and Applications 4 (1991), no. 4, 891-895.

[2] R. Dalmasso, Positive solutions of singular boundary value problems, Nonlinear Analysis. Theory, Methods \& Applications. An International Multidisciplinary Journal. Series A: Theory and Methods 27 (1996), no. 6, 645-652.

[3] W. Feng and J. R. L. Webb, Solvability of m-point boundary value problems with nonlinear growth, Journal of Mathematical Analysis and Applications 212 (1997), no. 2, 467-480.

[4] C. P. Gupta and S. I. Trofimchuk, Existence of a solution of a three-point boundary value problem and the spectral radius of a related linear operator, Nonlinear Analysis. Theory, Methods \& Applications. An International Multidisciplinary Journal. Series A: Theory and Methods 34 (1998), no. 4, 489-507.

[5] K. S. Ha and Y.-H. Lee, Existence of multiple positive solutions of singular boundary value problems, Nonlinear Analysis. Theory, Methods \& Applications. An International Multidisciplinary Journal. Series A: Theory and Methods 28 (1997), no. 8, 1429-1438.

[6] X. Liu, Nontrivial solutions of singular nonlinear m-point boundary value problems, Journal of Mathematical Analysis and Applications 284 (2003), no. 2, 576-590.

[7] R. Ma and N. Castaneda, Existence of solutions of nonlinear m-point boundary-value problems, Journal of Mathematical Analysis and Applications 256 (2001), no. 2, 556-567.

[8] J. R. L. Webb, Positive solutions of some three point boundary value problems via fixed point index theory, Nonlinear Analysis. Theory, Methods \& Applications. An International Multidisciplinary Journal. Series A: Theory and Methods 47 (2001), no. 7, 4319-4332.

[9] F. H. Wong, Existence of positive solutions of singular boundary value problems, Nonlinear Analysis. Theory, Methods \& Applications. An International Multidisciplinary Journal. Series A: Theory and Methods 21 (1993), no. 5, 397-406. 
[10] X. Xu, Multiplicity results for positive solutions of some semi-positone three-point boundary value problems, Journal of Mathematical Analysis and Applications 291 (2004), no. 2, 673-689.

[11] _ Positive solutions for singular m-point boundary value problems with positive parameter, Journal of Mathematical Analysis and Applications 291 (2004), no. 1, 352-367.

[12] X. Xu and J. Ma, A note on singular nonlinear boundary value problems, Journal of Mathematical Analysis and Applications 293 (2004), no. 1, 108-124.

[13] Z. Zhang and J. Wang, The upper and lower solution method for a class of singular nonlinear second order three-point boundary value problems, Journal of Computational and Applied Mathematics 147 (2002), no. 1, 41-52.

Xu Xian: Department of Mathematics, Xuzhou Normal University, Xuzhou, Jiangsu 221116, China E-mail address: xuxian68@163.com

Donal O’Regan: Department of Mathematics, National University of Ireland, Galway, University Road, Galway, Ireland

E-mail address: donal.oregan@nuigalway.ie 